\title{
Developing a pulsar-based time standard
}

\author{
G. Hobbs $\dagger$ \\ CSIRO Astronomy and Space Science, Australia Telescope National Facility, \\ PO Box 76, Epping NSW 1710, Australia
}

\begin{abstract}
We describe how observations of pulsars from the Parkes Pulsar Timing Array (PPTA) project have been used to develop a pulsar-based timescale. This is the first such timescale that has a precision comparable to uncertainties in international atomic timescales.
\end{abstract}

Keywords. time, pulsars: general

High precision timing observations of pulsars are being carried out at many observatories around the world. The main goal of these "pulsar timing array" (PTA) projects is to detect ultra-low frequency gravitational waves. As reviewed in this paper, these data sets can also be used to develop a pulsar-based time standard.

We have made use of observations from the Parkes radio telescope that were obtained as part of the Parkes Pulsar Timing Array (PPTA) project (Manchester et al. 2012). Initially the pulse times-of-arrival (ToAs) for each observation of each pulsar are determined. These ToAs are converted from the observatory time standard to a realisation of Terrestrial Time (TT). Barycentric arrival times are subsequently calculated and are compared with predictions of the arrival times using a model for the pulsar rotational and orbital parameters. The differences between the actual measurements and the predictions are known as the "pulsar timing residuals". This technique, known as "pulsar timing", is widely used in pulsar astronomy and is described in detail by Edwards, Hobbs \& Manchester (2006).

Various phenomena such as gravitational waves, unexplained timing irregularities or errors in terrestrial time standards will induce timing residuals. They can be distinguished by searching for correlations between the timing residuals of multiple pulsars. For instance, pulsar timing irregularities, glitch events or interstellar medium variations will lead to timing residuals that are uncorrelated between different pulsars. In contrast an error in the terrestrial time standard will lead to timing residuals that are identical for different pulsars (assuming that all pulsars have been observed over the same time span).

The incredible stability of millisecond pulsar rotation leads to the possibility of developing a time scale based on the pulsar rotation which is analogous to the free atomic scale, Échelle Atomique Libre (EAL). The Ensemble Pulsar Scale (EPS) can be used to detect fluctuations in atomic timescales and therefore can lead to a new realisation of TT.

Earlier attempts to develop a pulsar timescale have been made by Guinot \& Petit (1991), Petit \& Tavella (1996), Rodin (2008) and Rodin \& Chen (2011). Our new algorithm has been published in Hobbs et al. (2012) and is implemented as part of the TEMPO2 software package (Hobbs, Edwards \& Manchester 2006). This algorithm accounts for various features of the observations such as: 1) irregular sampling, 2) different data spans for different pulsars and 3) different fitting parameters for different pulsars. Our result is reproduced in Fig. 1. We successfully follow features known to affect the

$\dagger$ E-mail: george.hobbs@csiro.au 


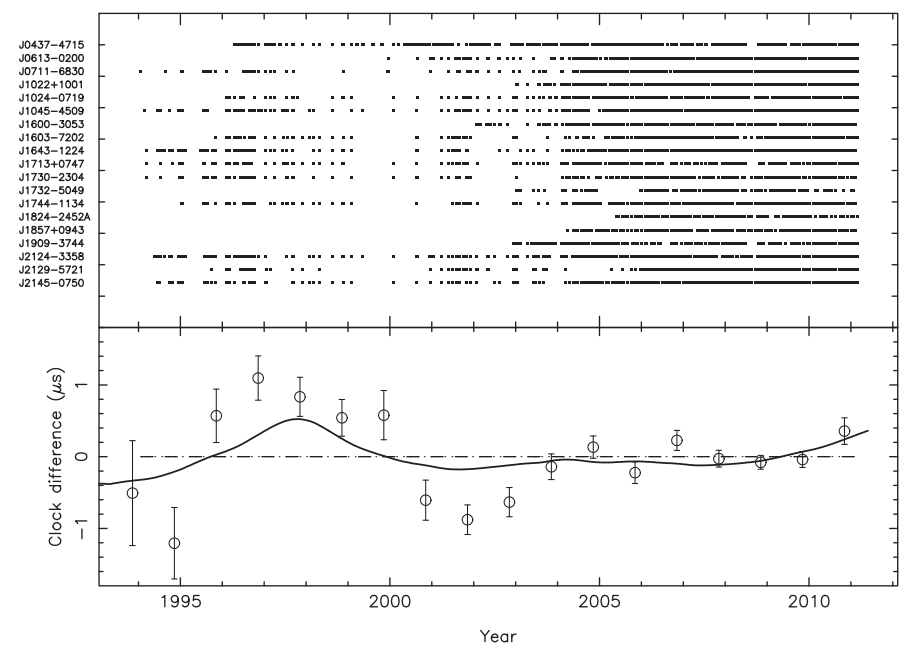

Figure 1. This figure is reproduced from Hobbs et al. (2012). The top panel shows the sampling for each of the pulsars in our sample. The lower panel shows the difference between the pulsar timescale and TT(TAI) as points with error bars. The solid line indicates the difference between TT(TAI) and TT(BIPM11) after a quadratic polynomial has been fitted and removed. Full details are available in Hobbs et al. (2012).

frequency of the International Atomic Timescale (TAI) and we find marginally significant differences between our pulsar time scale, TT(PPTA11), and TT(BIPM11).

This work is being continued by combining the Parkes observations with data from other observatories (see Hobbs et al. (2010) for a description of the International Pulsar Timing Array project). The new analysis will confirm or deny the tentative discrepancies between TT(PPTA11) and TT(BIPM11) whilst significantly improving the stability and precision of the pulsar scale. In the longer term it is expected that a future pulsar time scale will be combined with the best atomic timescale to give the world's most stable time scale that will be valid effectively forever.

\section{Acknowledgements}

The Parkes radio telescope is part of the Australia Telescope which is funded by the Commonwealth of Australia for operation as a National Facility managed by CSIRO.

\section{References}

Edwards, R., Hobbs, G., \& Manchester, R. 2006, MNRAS, 372, 1549

Guinot, B. \& Petit, G. 1991, A\&A, 248, 292

Hobbs, G., Edwards, R., \& Manchester, R. 2006, MNRAS, 369, 655

Hobbs, G., et al. 2010, CQGra, 27, 4013

Hobbs, G., et al. 2012, accepted by MNRAS (arXiv.1208.3560)

Manchester, R. N., et al. 2012, submitted to MNRAS

Petit, G. \& Tavella, P. 1996, A\& A, 308, 290

Rodin, A. 2008, MNRAS, 387, 1583

Rodin, A. \& Chen, D. 2011, Astronomy Reports, 55, 622 